\title{
Les éruptions volcaniques « explosives ": des grandes aux petites échelles
}

\author{
Claude Jaupart et Édouard Kaminski \\ Institut de Physique du Globe de Paris
}

\begin{abstract}
Les éruptions volcaniques riches en gaz, que l'on qualifie d'"explosives ", émettent de grandes quantités de gaz et de fragments de magma dans l'atmosphère. Elles peuvent prendre deux formes très différentes: une colonne légère pouvant s'élever jusqu'à plusieurs dizaines de kilomètres d'altitude, ou bien une coulée dense s'écoulant rapidement sur les pentes du volcan. Ces deux formes correspondent à de faibles différences de la quantité de gaz volcaniques dans l'éruption, qui sont elles-mêmes dues à de faibles différences de la taille des fragments de magma. Ces fragments, une fois trempés, deviennent des pierres ponces et des cendres que l'on ramasse facilement sur le sol. Ils contiennent des informations précieuses sur les phénomènes complexes qui se produisent sous terre avant l'éruption à la surface terrestre.
\end{abstract}

Les éruptions volcaniques sont difficiles à étudier et leur comportement reste mal compris, malgré plusieurs dizaines d'années de recherches. Dangereuses et destructrices, elles ne se prêtent pas à l'observation directe et ont la fâcheuse habitude de pulvériser les appareils de mesure qui se trouvent sur leur chemin. Elles sont responsables de grandes catastrophes humaines et de changements climatiques importants (mais réversibles). Pour ne citer qu'un exemple, l'éruption du volcan indonésien Tambora, en 1815, entraîna plusieurs années anormalement froides et de mauvaises récoltes jusqu'en Europe et en Amérique. L'année 1816 fût d'ailleurs qualifiée d'« année sans été ". Comme on le verra, ces éruptions peuvent changer brutalement de régime, et prévoir leur comportement serait difficile sans comprendre les mécanismes mis en jeu. Nous savons maintenant que ces changements intempestifs de régime sont dus à d'infimes variations des conditions physiques à la sortie du conduit éruptif.

Ce court article décrit brièvement les deux régimes volcaniques " explosifs " principaux et quelques points de physique essentiels pour comprendre ce qu'est une éruption volcanique. Nous parlerons aussi des fragments magmatiques qui sont transportés par les éruptions et que l'on retrouve sur le sol dans des dépôts qui atteignent parfois plusieurs centaines de mètres d'épaisseur. Notre objectif est de montrer que ces fragments ne sont pas dénués d'intérêt et que, bien au contraire, leurs propriétés et leur structure interne obéissent à une logique bien précise. En fait, ils contiennent des informations précieuses sur les éruptions et c'est en eux qu'il faut rechercher l'origine des formes différentes que peut prendre un écoulement volcanique. Ces fragments sont, en quelque sorte, à la fois les déterminants et les enregistreurs des conditions éruptives.

\section{Les deux régimes d'éruptions "explosives»}

\section{Les éruptions explosives}

Les magmas terrestres sont plus légers que les roches de l'écorce terrestre et sont propulsés vers le haut par la poussée d'Archimède. Ils contiennent des éléments volatils comme l'eau et le dioxyde de carbone. La solubilité de ces espèces est une fonction croissante de la pression. Dans la plupart des cas, ces éléments volatils sont dissous dans le magma aux fortes pressions qui règnent dans les réservoirs magmatiques. La baisse de pression qui résulte de l'ascension conduit inévitablement à l'apparition d'une phase gazeuse. La fraction massique de gaz augmente progressivement tout au long de la montée et, ajoutée à la dilatation, conduit à des fractions volumiques de gaz très élevées à la sortie. Pour une concentration de $1 \%$ en masse, une valeur tout à fait courante, la fraction volumique de gaz atteint $99 \%$ à la pression atmosphérique. Lors de la décompression, les relations entre les deux phases en présence, gaz et liquide, changent considérablement. À faible fraction volumique, le gaz est sous forme de bulles dispersées dans le magma : c'est une mousse magmatique qui s'écoule dans le conduit éruptif. Aux très fortes fractions volumiques qui prévalent à faible pression, une mousse n'est pas stable lorsqu'elle est cisaillée et se désagrège. C'est un jet de gaz portant des fragments (on pourrait dire des gouttes) de magma qui sort du conduit éruptif (figure 1). Le point essentiel qu'il faut retenir est que le mélange de gaz et de magma est dominé en masse par le magma, c'est-à-dire la phase dense, et dominé en volume par le gaz. Sa densité moyenne est bien plus élevée que celle d'un gaz et que celle de l'atmosphère, mais sa fraction volumique de gaz élevée lui confère le comportement d'un gaz.

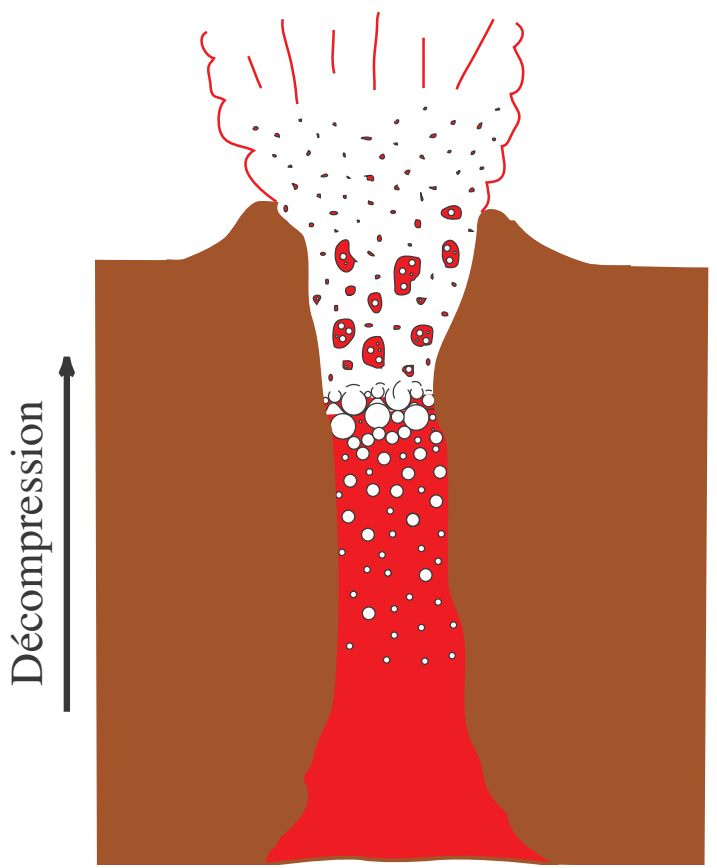

Figure 1. Schéma représentant les divers phénomènes dans un conduit volcanique. A forte pression, les éléments volatils sont dissous et c'est un liquide homogène qui s'écoule. Lorsque le seuil de solubilité est atteint, les volatils forment une phase gazeuse qui occupe un volume croissant lorsque la pression décroît. Le mélange volcanique passe ainsi d'un état de " mousse », tel que des bulles de gaz sont en suspension dans le liquide, à celui d'un jet de gaz portant des fragments de liquide. Le mécanisme responsable du changement d'état est appelé "fragmentation ». 
Une éruption sous la forme que nous venons de décrire est qualifiée d'“ explosive ». L'adjectif est impropre parce qu'il suggère un événement catastrophique bref, alors que ces éruptions peuvent se prolonger en régime quasi-permanent pendant plusieurs heures. On verra que le qualificatif d'explosif fait référence au phénomène de fragmentation.
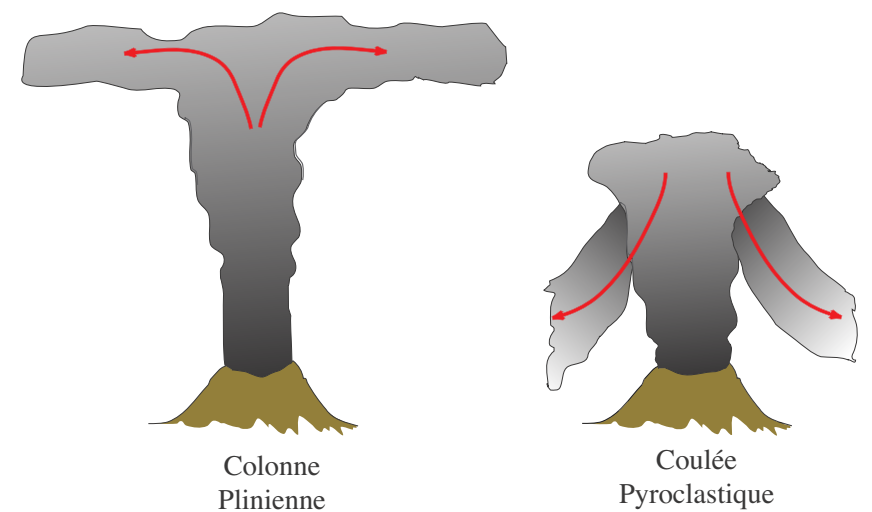

Figure 2. Les deux principaux régimes explosifs d'éruption volcanique, qui dépendent du comportement de la colonne éruptive et de l'intensité du phénomène d'entraînement (qui décrit l'ingestion d'air environnant par la turbulence du jet) : colonne Plinienne (à gauche) et écoulement pyroclastique (à droite).

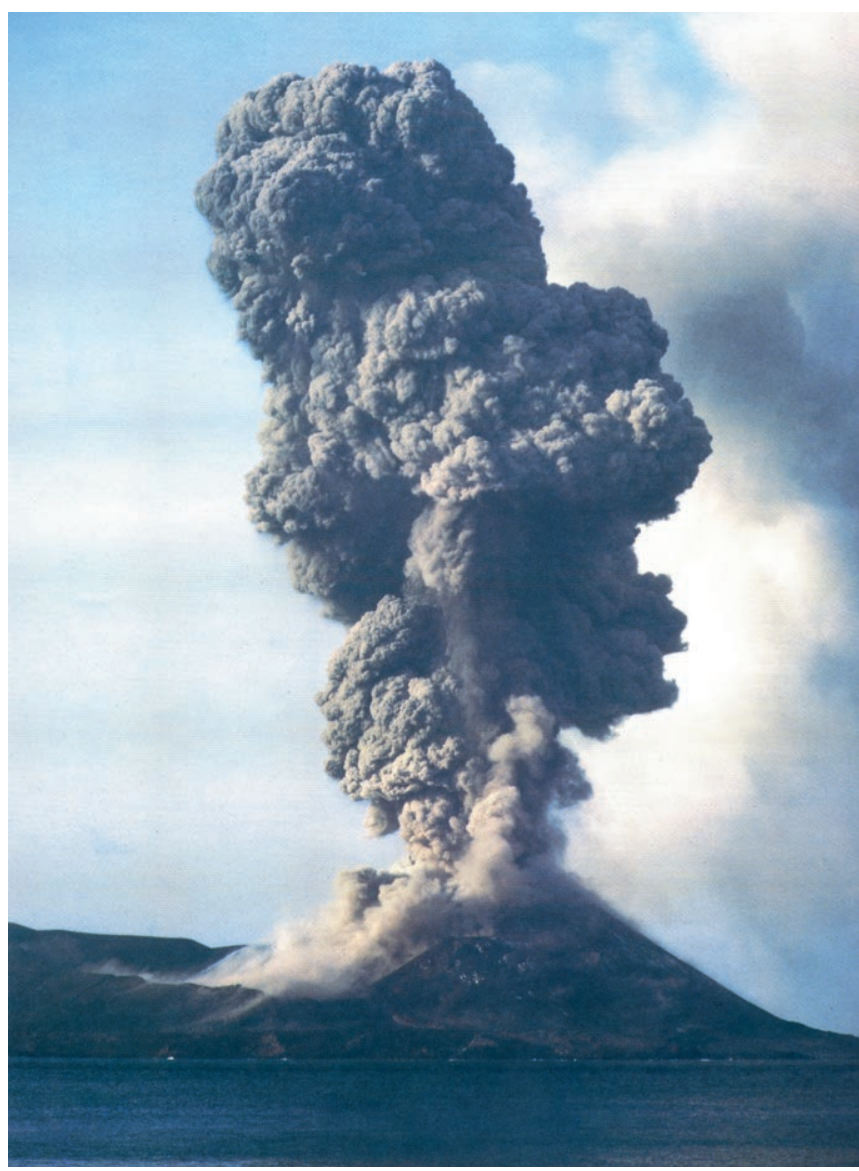

Figure 3. Une coulée pyroclastique du volcan indonésien Anak Krakatau, le descendant du gros volcan Krakatau qui dévasta le détroit de la Sonde en 1883. Le mélange de gaz et de fragments de magma ne s'élève qu'à environ 3 kilomètres et la coulée est visible sur le flanc gauche du volcan.

On connaît deux différents régimes explosifs principaux, appelés colonne Plinienne et coulée pyroclastique (figure 2). Dans le premier cas, le mélange de gaz et de fragments de magma ingère suffisamment d'air pour devenir plus léger que l'atmosphère : il est alors propulsé par poussée d'Archimède et forme une colonne atmosphérique qui peut s'élever à très haute altitude dans l'atmosphère (plusieurs dizaines de kilomètres, soit dans la stratosphère). Dans le deuxième cas, la colonne ne peut incorporer de grandes quantités d'air : le mélange reste plus lourd que l'air, ne monte qu'à quelques kilomètres au-dessus de la bouche éruptive et finit par retomber au sol, alimentant des coulées denses de fragments et de gaz qui dévalent les pentes du volcan (figure 3). Ces deux régimes se produisent pour des débits de magma semblables, mais ne représentent pas les mêmes dangers et n'ont pas le même impact sur l'environnement. Dans le cas Plinien, la colonne éruptive injecte les fragments de magma et les gaz volcaniques à très haute altitude, où les vents stratosphériques les dispersent sur de très grandes surfaces. Au sol, la conséquence est une pluie de cendres, désagréable et mauvaise pour la santé, mais non mortelle. Les coulées pyroclastiques sont bien plus dangereuses. Le mélange volcanique est concentré et canalisé par les vallées : il s'écoule à grande vitesse sur des hauteurs qui peuvent atteindre quelques centaines de mètres, dévastant tout sur son passage.

La très célèbre éruption du Vésuve en l'an 79 de notre ère connut ces deux régimes, et l'on sait rarement que ce sont des coulées pyroclastiques qui détruisirent Pompéi et Herculanum. Le géographe romain Pline l'Ancien était resté à Pompéi pendant l'éruption, par nonchalance et aussi à cause de sa corpulence. II se sentait capable de supporter la pluie de cendres de la phase initiale Plinienne, mais ne pouvait hélas savoir que l'éruption changerait brutalement de régime et se muerait en coulée pyroclastique. On peut se faire une idée précise de la brutalité du changement grâce aux fouilles archéologiques : les corps des habitants se trouvent au sommet des retombées Pliniennes, juste au-dessous du dépôt chaotique des coulées pyroclastiques.

Ces deux régimes sont bien définis, mais les choses sont plus compliquées en pratique. Les éruptions passent parfois de l'un à l'autre et sont souvent dans un régime intermédiaire où les deux types d'écoulement coexistent. C'est d'ailleurs ce qui rend ces éruptions particulièrement dangereuses, comme l'illustre bien l'exemple de Pline l'Ancien. En ce qui concerne la prévision et la surveillance d'un volcan en activité, un tel comportement ne facilite pas la tâche des volcanologues.

\section{Un peu de mécanique des jets et panaches turbulents}

Les éruptions explosives se décrivent comme des jetspanaches turbulents, pour lesquels la théorie est bien maitrisée et qui ont été abondamment étudiés en laboratoire. Compte tenu de la forte turbulence des écoulements volcaniques, gaz et fragments de magma ne se séparent quasiment pas et on peut traiter l'ensemble des deux comme une seule phase. On peut montrer que les profils radiaux de la vitesse, de la température et de la concentration de gaz sont décrits par la même fonction (une Gaussienne) indépendamment de l'altitude. On peut donc écrire les équations de conservation (masse, quantité de mouvement, énergie) pour des valeurs moyennes des variables en fonction de la seule variable altitude. Pour décrire la dynamique de l'écoulement, il faut suivre les variations du rayon du jet en fonction de l'altitude. Trois équations (masse, quantité de mouvement, énergie) pour trois variables (vitesse, densité, rayon) : le problème est bien posé. La solution dépend des valeurs initiales des trois variables : la fraction massique (ou volumique) de gaz, le rayon de l'évent et la vitesse verticale.

À la sortie de la bouche éruptive, le mélange est propulsé par sa quantité de mouvement. Sa masse volumique est calculée facilement avec la teneur en gaz: pour les quelques pour cents (en masse) de la plupart des volcans, celle-ci est 
de l'ordre de $200 \mathrm{~kg} \cdot \mathrm{m}^{-3}$. Cette valeur est très largement supérieure à celle de l'atmosphère, et le mélange volcanique devrait retomber vers le sol après quelques kilomètres d'ascension, à la manière d'un jet d'eau. En réalité, le caractère turbulent de l'écoulement lui permet d'aller beaucoup plus haut, car les tourbillons qui l'animent viennent incorporer de l'air environnant (on parle d'" entraînement "). Le contact avec les fragments de magma à haute température réchauffe l'air et fait fortement décroître sa densité. La fraction massique de gaz augmente, la densité du gaz diminue et par conséquent le mélange s'allège au fur et à mesure qu'il s'élève dans l'atmosphère. On peut envisager deux cas. Dans le premier, le mélange reste tout le temps plus dense que l'air. II décélère sous l'action de son poids et finit par retomber vers le sol : une coulée pyroclastique se forme. Dans le second cas, le jet incorpore beaucoup d'air et finit par devenir plus léger que l'atmosphère : propulsé par poussée d'Archimède, il se développe en colonne Plinienne à haute altitude (une vingtaine de kilomètres pour l'éruption de 1991 du Mont Pinatubo aux Philippines).

Pour comprendre comment les deux régimes dépendent des variables d'entrée (les valeurs initiales de la vitesse, de la densité et du rayon), on peut faire deux expériences simples par la pensée. On mesure l'intensité de la turbulence, et donc celle des phénomènes d'entraînement, par la vitesse verticale. Pour une vitesse donnée, plus le rayon de la bouche éruptive est grand, plus la surface externe du jet est faible en comparaison avec le volume, et donc plus la fraction d'air ingéré est faible en proportion. L'autre effet facile à imaginer est celui de la teneur en gaz du mélange. Pour une vitesse initiale et un rayon donnés, plus elle est faible à la sortie dans l'atmosphère, plus le mélange volcanique est dense et moins il sera susceptible de s'alléger suffisamment.

\section{Domaines d'existence des deux régimes éruptifs}

\section{Un diagramme simple}

Pour résoudre le problème pratique d'une éruption donnée, il faut connaître les valeurs initiales des trois variables : vitesse, densité et rayon. C'est difficile en pratique, surtout si l'on s'intéresse à des éruptions anciennes. En ce qui concerne la densité, le problème est facilement résolu. II suffit de déterminer la teneur en éléments volatils, le reste (le magma) étant parfaitement connu une fois que l'on a déterminé sa composition chimique. Les éléments volatils ne sont bien évidemment pas restés dans les dépôts. En revanche, on peut les retrouver dans d'infimes gouttelettes de magma piégées au sein de cristaux, que l'on appelle " inclusions vitreuses" parce qu'on les détecte et qu'on les étudie à faible température. Le piégeage se fait dans le réservoir du volcan, alors que les éléments volatils sont dissous dans le magma : les quantités dissoutes se mesurent facilement grâce aux techniques spectrométriques modernes. Une autre méthode repose sur les équilibres de phase : dans un magma, les minéraux qui précipitent et leurs compositions dépendent de la teneur en volatils.

Une fois les quantités de gaz volatils connus, restent à déterminer la vitesse et le rayon du jet volcanique. En fait, on peut reconstituer le flux de masse de l'éruption en utilisant le dépôt lui-même et un modèle théorique de formation, analogue aux modèles utilisés pour les dépôts sédimentaires. Le flux de masse est :

$$
\mathrm{Q}=\pi \mathrm{R}^{2} \rho \mathrm{W}
$$

où $\mathrm{R}$ est le rayon de la bouche éruptive, $\mathrm{W}$ la vitesse verticale moyenne et $\rho$ la densité du mélange. On peut donc déterminer les valeurs de deux variables : la teneur en gaz, qui permet de calculer la densité et le débit. Manque encore une variable ! L'astuce consiste à relier la sortie du conduit à l'écoulement en profondeur, pour lequel on connaît bien les phénomènes et les équations qui les décrivent quantitativement.

Si l'écoulement dans le conduit se faisait en régime laminaire contrôlé par la viscosité et compte tenu du fait que les pressions mises en jeu changent peu d'un système à l'autre, la vitesse de sortie devrait être approximativement une fonction décroissante de la viscosité. C'est bien ce qui est observé pour les coulées de lave : les coulées basaltiques (de viscosité égale à 10 Pa.s (Pascal-seconde), soit dix mille fois la viscosité de l'eau) sont émises à des vitesses nettement supérieures à celles de leurs analogues rhyolitiques (viscosité $\approx 10^{8} \mathrm{~Pa} . \mathrm{s}$ ), qui sont tellement lentes qu'on les qualifie de dômes de lave. Lorsque les magmas contiennent des volatils, ils se fragmentent avant la sortie et l'écoulement ne dépend plus des propriétés rhéologiques du magma. C'est un gaz chargé de particules qui monte dans le conduit et la viscosité n'est plus un facteur déterminant : l'écoulement est turbulent et le frottement ne dépend plus que de la vitesse et de la rugosité des parois. Dans un conduit aux bords verticaux, c'est-à-dire à section constante, la vitesse maximum est celle du son, qui prend des valeurs comprises entre 100 et 300 m.s ${ }^{-1}$ selon la teneur en volatils. On peut effectivement constater que les vitesses volcaniques sont de cet ordre de grandeur. Pour être plus précis, il faut introduire quelques détails. II est facile d'imaginer qu'un conduit volcanique n'est pas parfaitement vertical et que sa section varie probablement dans le temps sous l'effet même de l'éruption : par exemple, un cratère d'explosion s'évase vers le haut. Dans ce cas, le mélange peut dépasser la vitesse du son et subir une détente brutale à sa sortie dans l'atmosphère. Dans le cas d'une décompression libre, sans aucun effet de confinement, la vitesse est augmentée d'un facteur multiplicatif connu, dont la valeur est d'environ 2.

Ce résultat fournit l'information manquante et l'on peut représenter les régimes éruptifs en fonction de deux variables seulement : le flux de masse et la teneur en volatils (figure 4). Dans le diagramme ainsi obtenu, les éruptions à coulées pyroclastiques sont dans la moitié supérieure, correspondant aux gros conduits et aux (relativement) faibles teneurs en volatils. On notera que les données expérimentales situent systémati-

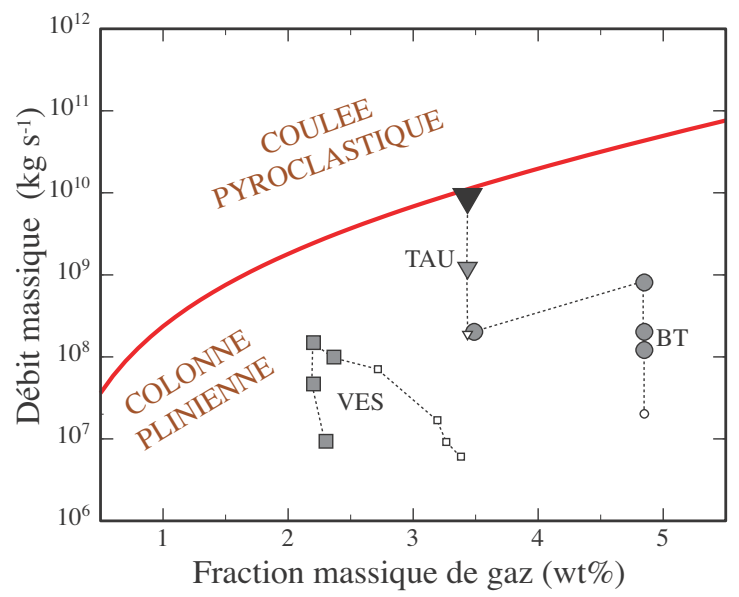

Figure 4. Diagramme montrant les domaines respectifs des deux régimes volcaniques explosifs tels qu'ils sont prédits par la théorie. On reporte aussi les valeurs connues des variables pour trois éruptions NES : éruption de $79 d u$ Vésuve ; TAU : éruption de Taupo en Nouvelle-Zélande ; BT : éruption de Bishop Tuff en Californie). Pour chaque éruption, les symboles sont les mêmes pour une éruption donnée et changent de taille et de couleur lorsque le régime éruptif change. Un petit symbole représente une phase Plinienne. Un symbole gris de taille moyenne indique un régime intermédiaire tel que coulée pyroclastique et colonne Plinienne coexistent. Un gros symbole noir désigne une phase de coulée pyroclastique. 
quement les régimes intermédiaires, et même la phase de coulée pyroclastique du Taupo, dans le domaine Plinien prédit par la théorie. Sur la foi de ces exemples, données et prédictions théoriques semblent en désaccord.

\section{Les caractéristiques des éruptions Pliniennes}

Grâce au modèle théorique, on peut prédire les caractéristiques d'une éruption, comme par exemple l'altitude atteinte par la colonne éruptive dans l'atmosphère et son régime (colonne Plinienne ou bien coulée pyroclastique), et aussi celles du dépôt qui en résulte. La validité de la théorie a été démontrée lors d'éruptions récentes, au Mont St Helens en 1980 ou bien encore au Mont Pinatubo en 1991 : les hauteurs des colonnes éruptives déterminées par observation directe étaient très proches des prédictions. Grâce au calcul, les caractéristiques d'un dépôt telles que la variation d'épaisseur et de taille des fragments en fonction de la distance à la bouche éruptive permettent de remonter à celles de l'éruption. Ceci est très utile, car un volcanologue ne peut se reposer sur les éruptions qui se produisent de son vivant : elles sont trop rares et se trouvent parfois dans des endroits difficiles d'accès. II est beaucoup plus fécond d'utiliser d'anciennes éruptions, dont le nombre est évidemment bien plus élevé. La figure 5 donne les caractéristiques d'une quarantaine d'éruptions anciennes et de quelques éruptions récentes. On peut être impressionné par l'énormité des dépôts (les plus gros pourraient recouvrir la France entière d'une couche d'un mètre d'épaisseur). On peut aussi remarquer que, avec des débits qui dépassent souvent $10^{8} \mathrm{~kg} \cdot \mathrm{s}^{-1}$ (cent millions de tonnes à la seconde!), ces dépôts se forment en quelques heures seulement. L'éruption de 1980 du Mont St Helens sur la côte Ouest des États-Unis d'Amérique est révélatrice. Traumatisante et sensationnelle lorsqu'elle s'est produite, elle est ramenée à sa juste place dans ce diagramme : celle d'une toute petite éruption qui ne laissera d'ailleurs quasiment aucun dépôt identifiable dans quelques centaines d'années.

\section{Quelques exemples concrets}

On peut maintenant comparer plus précisément prédictions et observations. La figure 4 montre plusieurs éruptions replacées dans le diagramme de régime. Toutes ces éruptions ont montré la même tendance, avec des débuts en régime Plinien et l'apparition de coulées pyroclastiques ensuite. Dans la majorité des cas, c'est en fait dans le régime intermédiaire, tel que colonne Plinienne et coulée pyroclastique coexistent, que se produisent les phases finales. Les phases de pure coulée pyroclastique sont rares. L'éruption de Taupo, en NouvelleZélande, fait exception avec une énorme phase terminale de coulée pyroclastique (laissant un dépôt appelé ignimbrite). On constate facilement qu'observations et prédictions ne sont pas en bon accord : les régimes intermédiaires observés se situent loin de la frontière théorique, et la phase de coulée pyroclastique du Taupo est dans le domaine Plinien. Où est l'erreur? Pour la trouver, il faut revenir au modèle physique, qui est bon et qui a été vérifié à de nombreuses reprises. L'erreur peut provenir d'une sous-estimation du flux de masse, ce qui est peu probable, ou de la teneur en gaz.

\section{Ponces et cendres}

\section{Deux phases gazeuses}

Les fragments portés par le jet volcanique ne sont pas denses : ils proviennent de la mousse magmatique qui s'écoule dans le conduit volcanique. Ce sont donc en fait des fragments poreux qui piègent des bulles de gaz. Le fait important est que le gaz magmatique se partage en deux composants bien distincts : l'un qui forme la phase continue de l'écoulement et qui porte les fragments, et l'autre qui reste

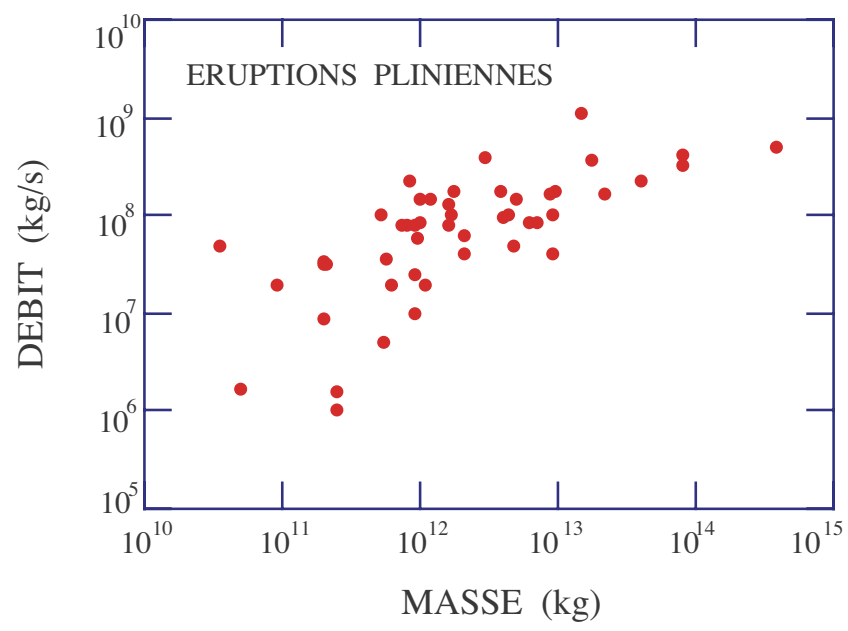

Figure 5. Les caractéristiques d'une cinquantaine d'éruptions Pliniennes. La célèbre éruption du Mont St Helens en 1980 est quasiment la plus petite et se situe en bas et à gauche.

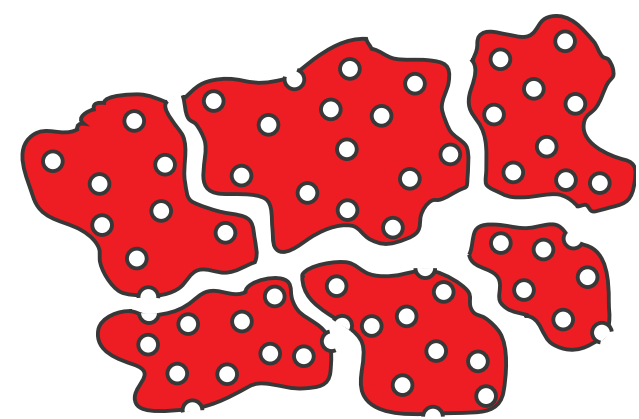

Fragmentation grossière

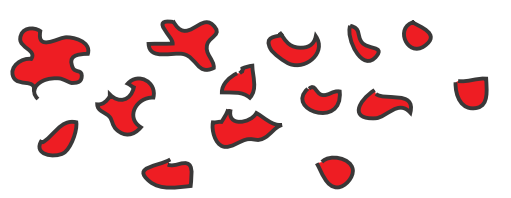

Figure 6. Les deux cas limites pour la fragmentation d'une mousse. En haut les fragments sont plus gros que les bulles : une proportion importante de bulles reste piégée à l'intérieur et peu de gaz est libéré. En bas, les fragments sont plus petits que les bulles: toutes les bulles ont éclate et tout le gaz est libéré. C'est le gaz libéré qui constitue la phase continue qui "porte »les fragments.

Fragmentation fine

piégé dans les fragments eux-mêmes. Bien évidemment, ces deux composants jouent des rôles très différents pour l'écoulement et la turbulence. Le gaz piégé est en quelque sorte « passif » et ne sert qu'à déterminer le poids des fragments. La masse de gaz " continu ", qui détermine le comportement de l'écoulement et l'intensité du mélange avec l'air ambiant, est inférieure à celle des éléments volatils contenus dans le magma à la source. On peut bien sûr imaginer que les fragments sont poreux et laissent échapper une partie de leur gaz, mais il est bien clair qu'une partie du gaz magmatique ne participe pas à l'écoulement général.

Cette discussion démontre qu'il faut séparer la phase gazeuse entre ses deux composants et déterminer la proportion de chacun. Un raisonnement simple montre que cette séparation dépend de la taille des fragments (figure 6). Partant d'une mousse de magma et de gaz que l'on découpe en morceaux, la seule manière de libérer tout le gaz est de pulvériser l'ensemble en faisant éclater toutes les bulles. Dans ce cas, les fragments ont une taille voisine de celle des bulles : figés puis trempés, ils deviennent des cendres. En fait, la " découpe » de la mousse n'est pas aussi parfaite et engendre de gros fragments : ceux-ci sont pleins de bulles et, trempés, deviennent des pierres ponces. On peut déterminer la fraction de gaz qui est libérée à la fragmentation : il suffit de calculer le nombre de bulles intersectées par la surface de fracturation 
(figure 6). On comprend facilement que cette fraction dépend du rapport entre la taille du fragment et le rayon des bulles qu'il contient : plus le fragment est gros, plus elle est faible.

\section{La taille des fragments de magma}

Dans la réalité, les fragments n'ont pas tous la même taille, mais appartiennent à une population. On peut déterminer directement la distribution des tailles à partir d'échantillons prélevés dans les dépôts, par tamisage. L'opération est longue et fastidieuse, mais a été réalisée en de nombreux endroits. On représente en fonction de $\mathrm{R}$ la distribution par la proportion $N(R)$ de fragments dont la taille est supérieure à une valeur $R$. On trouve que :

$$
N(R)=C R^{-D}
$$

où $\mathrm{D}$ est un exposant caractéristique et $\mathrm{C}$ une constante de normalisation (figure 7). Plus $\mathrm{D}$ est grand, plus la population est dominée par les petits fragments. On utilise couramment une " unité de tamisage », notée $\Phi$, telle que la maille de la grille du tamis vaut $2^{-\Phi}$ en millimètres. Les tamis sont fabriqués avec des tailles qui varient d'un facteur 2 et $\Phi$ représente la maille moyenne. La figure 7 montre la population des fragments de l'éruption du volcan Askja en Islande. On peut constater que la distribution est bien régulière sur une très large gamme de valeurs : près de cinq ordres de grandeur! L'exposant prend une valeur de 3 , ce qui est assez inhabituel et riche d'enseignements sur le phénomène de fragmentation lui-même, comme on le verra plus loin.

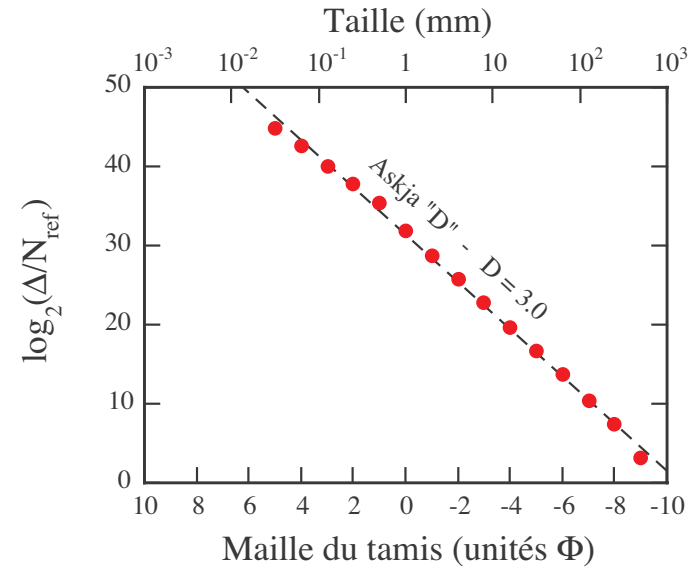

Figure 7. Distribution des tailles de fragments de l'éruption Plinienne du volcan Askja, Islande. Les tailles sont représentées avec deux unités : la maille de grille des tamis et le $\mathrm{mm}$. Le nombre de fragments pour chaque valeur $\Phi$ de l'unité de tamisage, noté $\Delta(\Phi)$, est normalisé par une constante multiplicative. Son logarithme est reporté en fonction de $\Phi$ et la pente de la droite donne l'exposant D de la loi de puissance.

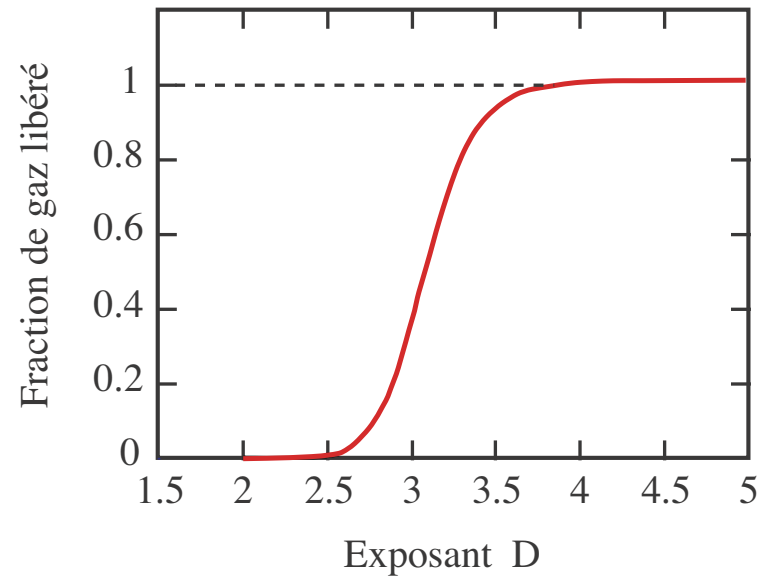

Figure 8. Fraction de gaz libéré par la fragmentation du magma en fonction de l'exposant $D$ de la distribution de taille.
Une fois la distribution de taille connue, on peut calculer la fraction de gaz libérée à la fragmentation en fonction de la taille des bulles et de la valeur de l'exposant $D$ (figure 8). Le calcul théorique est assez facile (voir les références bibliographiques) parce que les bulles sont bien plus petites que la plupart des fragments. Le calcul permet en outre de démontrer que le résultat n'est pas sensible à la taille des bulles et à la dispersion des tailles dans la gamme des valeurs observées. On peut remarquer que la fraction libérée varie fortement lorsque l'exposant D prend des valeurs voisines de 3 . Cette constatation prend tout son sens lorsqu'on la rapproche d'une deuxième observation : c'est précisément autour de 3 que se situent les valeurs de $\mathrm{D}$ des populations volcaniques.

\section{Les populations de ponces et de cendres}

On constate que les valeurs de l'exposant $D$ sont systématiquement plus élevées pour les dépôts Pliniens que pour ceux des coulées pyroclastiques (figure 9). La conséquence est que la fraction de gaz libérée est plus importante pour les premiers que pour les secondes, ce qui est en accord avec ce que l'on sait des deux régimes. On voit ici le lien étroit qui existe entre l'écoulement à grande échelle (plusieurs dizaines de kilomètres) et les tout petits fragments qu'il transporte (la plupart ne dépassent pas quelques centimètres). La signification de ce résultat apparaît clairement en se reportant ensuite à la figure 4. Dans celle-ci, les calculs se réfèrent à la quantité de gaz porteur, alors que les mesures correspondent à la quantité totale de gaz. Pour une comparaison avec les courbes théoriques, il faut mettre en abscisse la quantité de gaz porteur, ce qui revient à translater vers la gauche les points expérimentaux de la figure 4 . On obtient alors un bon accord entre prédictions et mesures.

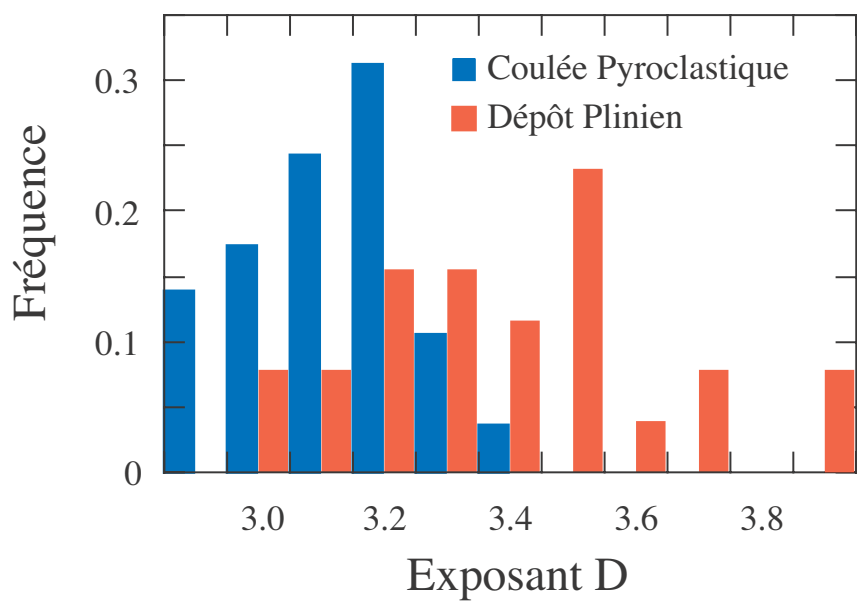

Figure 9. Valeurs de l'exposant $D$ de la distribution des tailles des fragments d'un grand nombre d'éruptions. On note que les valeurs de D sont systématiquement plus petites pour les coulées pyroclastiques que pour les colonnes Pliniennes. En se reportant à la figure 8, on en déduit que la proportion de gaz "porteur ", c'est-à-dire de la phase continue qui charrie les fragments de magma dans la colonne éruptive, est plus faible pour les coulées pyroclastiques que pour les colonnes Pliniennes.

\section{Le phénomène de fragmentation}

Le phénomène de fragmentation revêt une importance toute particulière, car c'est lui qui détermine le régime de l'écoulement, aussi bien dans le conduit éruptif sous la surface que dans l'atmosphère. Sous la surface, c'est lui qui marque le passage entre l'écoulement laminaire du magma chargé de bulles et l'écoulement turbulent de gaz chargé de fragments. Après la sortie dans l'atmosphère, c'est lui qui détermine si l'éruption se développe en colonne Plinienne ou en coulée pyroclastique. 


\section{Un seul épisode de fragmentation ?}

On peut postuler que la fragmentation est un phénomène rapide aux effets immédiats et définitifs. C'est l'hypothèse qui a été faite jusqu'à présent dans les calculs : concrètement, la fragmentation est associée à un seuil bien défini, par exemple une fraction volumique de gaz donnée, telle que la mousse magmatique n'est plus stable. On peut reproduire le phénomène en laboratoire. Dans ce cas, bien étudié dans de multiples contextes (explosions, impacts, etc...), la distribution de taille des fragments suit bien une loi de puissance, mais l'exposant $D$ est inférieur à 3 . Cette borne supérieure découle de l'hypothèse d'un unique phénomène agissant de la même manière à toutes les échelles et conservant la masse totale. On pourrait se demander si cette hypothèse est valable pour une mousse. Deux types d'expériences ont été menées à cet effet: l'une sur les ponces elles-mêmes, cassées par l'impact d'une grosse masse, et l'autre sur un véritable magma décomprimé dans une enceinte spéciale. Dans les deux cas, la distribution de taille est telle que $D \approx 2,5$, une valeur retrouvée pour de nombreuses populations d'objets dans la nature.

On doit naturellement se demander pourquoi les valeurs de $D$ des populations volcaniques sont supérieures à 2,5 et comment elles peuvent dépasser la valeur-limite de 3. Pour comprendre, il suffit de se rappeler ce qui préside aux valeurs "normales » : un phénomène qui affecte de la même manière tous les objets quelle que soit leur taille. En d'autres termes, on doit rechercher un mécanisme dont l'efficacité et la probabilité dépendent de la taille. Si l'on se réfère à la figure 8 , qui montre la fraction volumique de gaz libérée à la fragmentation, on constate que celle-ci est faible pour des valeurs de D inférieures à 2,5, voire à 3 . En fait, la courbe illustre parfaitement la très importante différence entre des populations avec des valeurs de $D$ supérieures ou inférieures à 3 . Pour $D \approx 2,5$, la fraction de gaz est faible : les fragments sont quasiment jointifs et rentrent fréquemment en collision les uns avec les autres. II est évident que la probabilité de collision pour un fragment dépend de sa taille (la section « efficace » bien connue dans d'autres domaines de la physique). En introduisant une probabilité de fragmentation qui augmente avec la taille, on obtient des populations dont les valeurs de D peuvent dépasser la valeur-seuil de 3 .

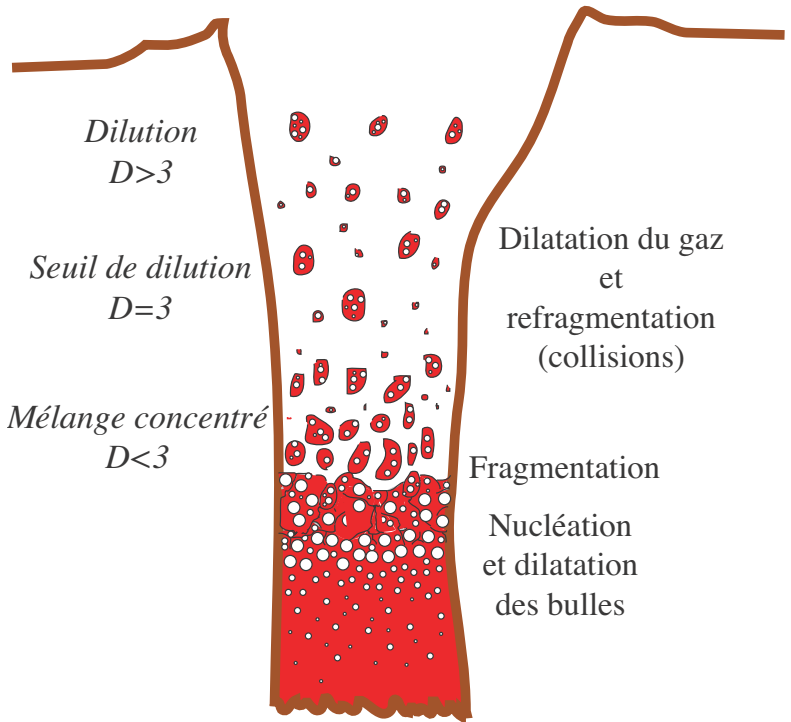

Figure 10. Schéma montrant la séquence des phénomènes affectant les fragments et le mélange éruptif dans un conduit. On peut suivre la séquence grâce aux valeurs de D. Celle-ci vaut 2,5-2,6 à la fragmentation et augmente progressivement à cause des multiples collisions que subissent les fragments dans l'écoulement turbulent. Lorsque la valeur de D est grande, la fraction volumique de gaz dans le mélange est élevée et les collisions sont rares : la population de fragments a atteint son état final.

\section{Synthèse}

Ces considérations montrent que les fragments pyroclastiques enregistrent toute une série d'événements. Ils nous apportent de précieux renseignements sur l'écoulement dans un conduit volcanique, qu'il serait difficile d'obtenir directement ! On peut désormais se représenter une éruption explosive comme un écoulement turbulent chargé de fragments dont les collisions dépendent de l'intensité de la turbulence, de la taille des fragments et de la fraction de gaz. Cette dernière variable augmente sous deux effets bien distincts : d'un côté la décompression et la dilatation qui en découle, de l'autre côté la refragmentation qui amène à des fragments plus petits et qui libère ainsi du gaz piégé. On peut montrer que les deux effets sont d'importance quasi-égale.

La figure 10 décrit un schéma des phénomènes dans le conduit volcanique avec, d'un côté la population de fragments (caractérisée par ses valeurs de D) et de l'autre les régimes d'écoulement et de fragmentation. Au bout d'un certain temps, pour une baisse de pression suffisamment importante et pour une valeur de D suffisamment élevée, la fraction volumique de gaz est tellement élevée que les collisions entre fragments deviennent rares et la valeur de D n'évolue plus.

\section{Conclusion}

Les éruptions volcaniques révèlent une partie de leur mystère grâce aux échantillons qu'ils portent et déposent. Ceux$\mathrm{ci}$, les pierres ponces que nous connaissons bien depuis l'Antiquité, sont en quelque sorte des traceurs des conditions volcaniques. D'un point de vue plus général, on doit être frappé par le fait que ce sont de tout petits objets qui déterminent le devenir d'une colonne éruptive se développant sur des dizaines de kilomètres d'altitude. On pourrait décrire dans un article plus long toute l'information que l'on peut tirer des ponces et des cendres : décomprimées et trempées dans la colonne atmosphérique, elles donnent des informations sur le taux de refroidissement qu'elles subissent, et donc sur les caractéristiques de l'écoulement (parmi lesquelles la vitesse).

Les deux régimes décrits ici sont des cas limites et nous butons à l'heure actuelle sur les régimes intermédiaires. D'autres phénomènes restent mal compris. Par exemple, certains dépôts assez fins (quelques mètres d'épaisseur) montrent des litages remarquables, semblables à ceux des courants de turbidité, d'autres suggèrent des phases violentes appelées « déferlantes », que nous ne savons pas encore expliquer. Ces complications illustrent à leur manière le problème des grandes différences d'échelle. Un courant puissant mais bref, n'est sans doute qu'un avatar sans grande signification pour la colonne éruptive toute entière, qu'il est raisonnable de négliger dans la théorie. À la petite échelle de l'affleurement et donc à celle des êtres humains, ce même courant peut être celui qui dévasta Pompéi et tua Pline l'Ancien.

Ce court article illustre le travail d'équilibriste du volcanologue moderne, qui doit jongler avec le travail de terrain, la mécanique des fluides et les phénomènes de fragmentation. II illustre aussi le nécessaire aller et retour entre théorie et observations naturelles. La valeur d'une théorie ne réside pas uniquement dans son côté quantitatif, mais aussi dans sa capacité à orienter les stratégies de mesure sur le terrain.

\section{Bibliographie (courte)}

- Claude Jaupart, Les volcans, collection « Dominos », Flammarion (1998).

- Divers auteurs (sous la direction de H.C. Nataf et J. Sommeria), La physique et la terre, Belin - CNRS Editions (2000).

- Divers auteurs, Encyclopedia of Volcanology, Academic Press (2000) 